\title{
POLY(VINYLPYRROLIDONE)-CHITOSAN HYDROGELS AS MATRICES FOR CONTROLLED DRUG RELEASE
}

\author{
ALINA MIRELA IPATE, ${ }^{*}$ DIANA SERBEZEANU, ${ }^{*}$ ALEXANDRA BARGAN, ${ }^{*}$ \\ CORNELIU HAMCIUC, ${ }^{*}$ LĂCRĂMIOARA OCHIUZ ${ }^{* *}$ and SIMONA GHERMAN ${ }^{* *}$ \\ " "Petru Poni” Institute of Macromolecular Chemistry, 41A, Gr. Ghica Voda Alley, \\ 700487, Iasi, Romania \\ ** "Grigore T. Popa” University of Medicine and Pharmacy, Faculty of Pharmacy, 16, University Str., \\ 700115, Iasi, Romania \\ \Corresponding author: Diana Serbezeanu, diana.serbezeanu@icmpp.ro
}

Received September 7, 2020

In this study, hydrogels based on poly(vinylpyrrolidone) and chitosan, derived from different mixing ratios of poly(vinylpyrrolidone)/chitosan, were synthesized by the freeze-drying technique with the aim of obtaining new systems that could be used to release tetracycline hydrochloride (TH). Freeze-drying methods were also used to prepare the hydrogels containing TH. The hydrogels were characterized using Fourier transform infrared spectroscopy and scanning electron microscopy, as well as in terms of dynamic water vapour sorption capacity. The poly(vinylpyrrolidone)/chitosan hydrogels were evaluated with regard to the release of $\mathrm{TH}$. The release profiles of $\mathrm{TH}$ from the poly(vinylpyrrolidone)/chitosan hydrogels depended on the chitosan content. It could be seen that if the concentration of chitosan was too high, the release was delayed and not fully achieved, because the release of the drug was prevented by the crystalline areas of the chitosan. According to the release study, the drug release mechanism of the poly(vinylpyrrolidone)/chitosan hydrogels loaded with TH perfectly fitted the Higuchi and the Korsmeyer-Peppas models. The highest value of water vapor sorption capacity was obtained for the hydrogel with the poly(vinylpyrrolidone)/chitosan ratio of 50/50. PVP-CS loaded with $3 \mathrm{wt} \% \mathrm{TH}$ may potentially be used for the controlled delivery of tetracycline to treat oral infections.

Keywords: poly(vinylpyrrolidone), chitosan, tetracycline, hydrogels, release study

\section{INTRODUCTION}

Poly(vinylpyrrolidone) (PVP) is a synthetic polymer that has been widely utilized in different applications due to its biocompatibility and non- toxicity, its capability to form stable association compounds and complexes with active substances, its affinity for hydrophilic and hydrophobic surfaces, thereby providing lyoprotectant action. ${ }^{1-3}$ PVP is widely used in medicine and cosmetics, for pharmaceutical and biomedical applications. ${ }^{3,4}$ PVP has the capacity to hold all the components of a drug together until the tablet reaches the stomach and then is rapidly dissolved or disintegrated to release the drug.

Chitosan (CS) is a natural cationic copolymer composed of $\beta$-(1,4)-2-acetamido-2-deoxy-dglucose and $\beta$-(1,4)-2-amino-2-deoxy-d-glucose units produced by the deacetylation of chitin. Because of the high ratio of acetylated groups, its rigid structure and poor solubility, chitin is not readily applicable. So, the partial deacetylation of chitin leads to chitosan. The applicability of chitosan in several fields, such as agriculture, waste treatment, food, textile and pharmaceutical industries, as well as cosmetic development and biomaterials, is due to a sum of characteristics, such as healing, antimicrobial, antifungal and chelating properties. However, the use of chitosan for biomedical applications has been limited by its relatively lower elongation, low toughness, poor hydrolytic stability, high degree of swelling in water, relatively poor water vapor barrier characteristics as well as being relatively expensive compared to conventional plastics. ${ }^{5,6} \mathrm{In}$ order to overcome these scientific and technological challenges and expand its range of applications, various strategies have been Cellulose Chem. Technol., 55 (1-2), 63-73(2021) 
explored. For example, synthetic polymers have been blended with chitosan to generate chitosan films with improved mechanical and functional properties. $^{7,8}$ It has been reported that chitosan and PVP are miscible polymers. ${ }^{9}$ This observation may be due to the hydrogen bond formation between the carbonyl groups of the PVP pyrrolidone rings and the amino/hydroxyl groups present in chitosan. ${ }^{10}$ The interaction between chitosan and PVP allows for the beneficial properties of both polymers to be combined in order to generate novel biocompatible CS-PVP blends, with improved physicochemical and mechanical properties.

Mucosal administration has the advantage of the rapid entry of the drug into the systemic circulation, which leads to increased bioavailability. ${ }^{11}$ Hydrogels offer excellent potential as oral therapeutic systems, due to the inherent biocompatibility of its component materials, potential use of both natural and synthetic materials and their adaptable properties. ${ }^{12}$ Hydrogels are hydrophilic viscoelastic materials formed from a threedimensional polymer network, with physical and/or chemical cross-links, that absorb and retain large amounts of water or biological fluids and swell, keeping their 3D structure. ${ }^{13}$ The high water content provides an environment for the diffusion of oxygen, nutrients and other small molecules, which are critical for cell growth and proliferation. ${ }^{14}$ Hydrogels maintain their mechanical strength and elasticity, remaining insoluble in the environment, thereby exhibiting similar physical properties with those of living tissues. ${ }^{15}$ Due to the property of hydrogels to mimic tissues, they have been used as matrices for local administration of drugs, including antibiotics. ${ }^{16}$ By incorporating the drug into hydrogels, the therapeutically active molecules are protected from premature degradation, thus improving the efficacy of the drug and minimizing side effects. ${ }^{17}$

Due to the increasing resistance of microorganisms to antibiotics, in recent years, research has been focused on their local administration to the infected site, thus minimizing the conventional dose and the frequency of administration. ${ }^{18}$ Until now, a large number of local drug delivery systems and devices have been proposed for oral and dental applications, including fibers, tapes, films, gels, sponges, microparticles, and so on. ${ }^{19-22}$ Applying drug loaded hydrogels to the oral mucosa may facilitate controlled drug delivery.

Tetracycline hydrochloride (TH) is a bacteriostatic antibiotic with a broad spectrum of action, which inhibits both Gram-negative and positive organisms. ${ }^{23,24}$ It exhibits antiproteolytic and anticollagenase properties, and it is also commonly used to treat periodontal disease. ${ }^{25}$

The hydrogels presented in this paper were prepared on the basis of the freeze-drying method using PVP and chitosan. This method avoids the use of toxic agents in the synthesis of hydrogels. Chitosan was selected as polymeric matrix due to its proven antimicrobial activity. ${ }^{26}$ The porous chitosan/PVP hydrogels were further investigated as scaffolds for controlled release of $\mathrm{TH}$, which should be effective in treating bacterial infections and oral candidiasis.

\section{EXPERIMENTAL}

\section{Materials}

Poly(vinylpyrrolidone) (PVP) (approximate molecular weight $\mathrm{Mw}=40000$; product number PVP40), chitosan (low molecular weight with $75 \%$ deacetylation), glacial acetic acid, and tetracycline were purchased from Sigma-Aldrich (St. Louis, USA). All other reagents were used as received from commercial sources or were purified by standard methods.

\section{Preparation of hydrogels}

A $10 \mathrm{wt} \%$ solution of PVP disolved in double distilled water was prepared by constant stirring, at room temperature for $24 \mathrm{~h}$. Separately, $1 \mathrm{wt} \%$ solution of CS was obtained by dissolving CS in a $0.1 \mathrm{M}$ acetic acid solution at room temperature, under stirring for 24 h. The two solutions were mixed together at room temperature for 6 hours, using different PVP-CS ratios, in order to obtain the blank samples denoted as PVPCS-1, PVP-CS-2 and PVP-CS-3. The drug loaded samples were prepared by adding tetracycline hydrochloride ( $3 \mathrm{wt} \%$ ) to these blank solutins to give the samples denoted as PVP-CS-1', PVP-CS-2' and PVP-CS-3' (Table 1). Four freeze-thaw cycles, alternating from freezing at $-20{ }^{\circ} \mathrm{C}$ for $24 \mathrm{~h}$ to thawing at room temperature over a period of $24 \mathrm{~h}$, were achieved on the resultant solutions. All the obtained hydrogels were freeze-dried over $48 \mathrm{~h}$ at $-46{ }^{\circ} \mathrm{C}$ and were stored in a fridge at $5{ }^{\circ} \mathrm{C}$. The composition of the hydrogels is listed in Table 1.

\section{Methods \\ Attenuated total reflection Fourier transform IR (ATR-FTIR) spectroscopy}

FTIR spectra of the samples were obtained using a BioRad FTS 1350 FTIR spectrometer, equipped with a Specac "Golden Gate" ATR accessory. A LUMOS 
Fourier Transform Infrared (FTIR) Microscope (Bruker Optik GmbH, Ettlingen, Germany), equipped with an attenuated total reflection (ATR) device, was used to record the scans between 4000 and $600 \mathrm{~cm}^{-1}$ at a resolution of $4 \mathrm{~cm}^{-1}$.

Table 1

Composition of blank and drug loaded PVP-CS hydrogels

\begin{tabular}{lccc}
\hline Sample & $\begin{array}{c}\text { PVP } \\
(\mathrm{wt} \%)\end{array}$ & $\begin{array}{c}\text { CS } \\
(\mathrm{wt} \%)\end{array}$ & $\begin{array}{c}\text { Tetracycline } \\
(\mathrm{wt} \%)\end{array}$ \\
\hline PVP-CS-1 & 80 & 20 & - \\
PVP-CS-1' $^{\prime}$ & 80 & 20 & 3 \\
PVP-CS-2 & 50 & 50 & - \\
PVP-CS-2' & 50 & 50 & 3 \\
PVP-CS-3 & 20 & 80 & - \\
PVP-CS-3' & 20 & 80 & 3 \\
\hline
\end{tabular}

Morphological characterization via scanning electron microscopy (SEM)

Scanning electron microscopy (SEM) was performed on a TESLA BS 301 microscope, at $20 \mathrm{kV}$, with a magnification of 380-3600. The images were recorded on film surfaces deposited on gold supports and coated by sputtering with thin gold films using an EK 3135 EMITECH device.

\section{Dynamic water vapor sorption capacity}

Dynamic water vapor sorption capacity for the samples was determined using the fully automated Intelligent Gravimetric Analyzer (IGA) (Hiden Analytical, Warrington, UK), and generated data were processed using the IGAsorp specific software package (Hiden Analytical, Warrington, UK). Before sorption measurement, the samples were dried at $25{ }^{\circ} \mathrm{C}$, in flowing nitrogen $(250 \mathrm{~mL} / \mathrm{min})$ until the weight of the sample was constant at relative humidity $(\mathrm{RH})<1 \%$. Then, the relative humidity was gradually increased from 0 to $90 \%$, at a rate of $10 \% \mathrm{RH}$ per h. Afterwards, the $\mathrm{RH}$ of the equipment was decreased in a similar pattern and the desorption curves were recorded.

\section{Estimation of drug loading and entrapment efficiency}

The quantitative determination of tetracycline hydrochloride $(\mathrm{TH})$ was performed using an earlier reported spectrophotometric method (SPECORD 210 PLUS-223F2042C) based on absorbance measurement. In order to determine the wavelength at which it absorbs the pure drug $(\mathrm{TH})$, a dilute solution of $\mathrm{TH}$ in phosphate buffer of $\mathrm{pH} 7.4$ was prepared and the absorption spectrum in the UV-VIS domain was recorded. After this determination, the calibration curve for TH was drawn in phosphate buffer $(\mathrm{pH} 7.4)$ in the concentration range of $5-40 \mu \mathrm{g} / \mathrm{mL}$, for which the absorbance values were recorded at the set wavelength. In order to establish the loading of the drug in the polymer matrix and the efficiency of its loading, samples were taken from three different places of each hydrogel, which were introduced into $25 \mathrm{~mL}$ flasks containing phosphate buffer of $\mathrm{pH}$ 7.4. The solutions were introduced into an ultrasonic bath for 2 hours at $37{ }^{\circ} \mathrm{C}$, then centrifuged, and samples were taken from the supernatant, which were diluted to a concentration in the study area and then analyzed spectrophotometrically. The measured absorbance values were identified on the calibration curve and, by interpolation, the $\mathrm{TH}$ concentrations in each polymer matrix were accurately determined. The load capacity (LC) and load efficiency (EE) results for tetracycline hydrochloride $(\mathrm{TH})$ were calculated using the equations:

$L C(\%)=\left(\frac{M_{\text {actual TH }}}{M_{\text {gample }}}\right) \cdot 100$

where LC - loading capacity of TH in hydrogels; $\mathrm{M}_{\text {actual TH }}$ - actual amount of TH loaded in hydrogel sample; $\mathbf{M}_{\text {sample }}$ - actual amount of hydrogel sample;

$\boldsymbol{E} \boldsymbol{E}(\%)=\left(\frac{\boldsymbol{M}_{\text {actual TH }}}{\boldsymbol{M}_{\text {tearetieal TH }}}\right) \cdot \mathbf{1 0 0}$

where $\mathrm{EE}$ - entrapment efficiency, \%; $\mathrm{M}_{\text {actual }} \mathrm{TH}-$ actual amount of $\mathrm{TH}$ loaded in hydrogel; $\mathrm{M}_{\text {theoretical } \mathrm{TH}^{-}}$ theoretical amount of $\mathrm{TH}$ added in the hydrogel preparation.

\section{In vitro drug release studies}

This study was carried out using a phosphate buffer release medium ( $\mathrm{pH} 7.4),{ }^{27}$ according to the following experimental protocol: dissolution medium: $500 \mathrm{~mL}$ of phosphate buffer solution, apparatus: 2 paddles; bath temperature: $37^{\circ} \mathrm{C} \pm 0.5^{\circ} \mathrm{C}$; rotation speed: $100 \mathrm{rpm}$; sampling interval was set at every 15 min during the study period $(1 \mathrm{~h})$. Each sample containing the equivalent of $10 \mathrm{mg}$ TH was weighed and was placed directly in the dissolution bath with $500 \mathrm{~mL}$ of phosphate buffer solution. The drug releasing medium (3 $\mathrm{mL})$ was withdrawn at various time intervals and replaced with the same volume of medium. The amount of tetracycline hydrochloride released was determined using the spectrophotometric method described above. 


\section{Analysis of in vitro drug release kinetics}

In order to predict and correlate the in vitro release behavior of tetracycline hydrochloride within the hydrogel system, suitable mathematical models were used. The experimental data obtained from the in vitro drug release studies were assessed using three prediction models: zero-order, first-order, and Korsmeyer-Peppas release kinetics. ${ }^{28}$ Data fitting was performed by linear and nonlinear regression using Matlab 7.1. Data were presented as mean \pm standard deviation and differences between data sets were considered statistically significant when $\mathrm{p}<0.05$. The Akaike Information Criterion (AIC) and the correlation coefficient $\left(\mathrm{R}^{2}\right)$ were the criteria for selecting the model that reliably described the release profile of the formulation, with the value of $\mathrm{R}^{2}$ close to 1 , and low AIC values. ${ }^{29,30}$

\section{RESULTS AND DISCUSSION} Structural and morphological characterization

The FTIR spectra of the freeze-dried hydrogel samples are presented in Figure 1. The peaks at 2974, 2830, 1647 and $1560 \mathrm{~cm}^{-1}$ could be assigned to the stretching vibration of $\mathrm{C}-\mathrm{H}$ (asym. and sym.), $\mathrm{C}=\mathrm{O}$ and $\mathrm{C}-\mathrm{N}$ groups, respectively. The signals of amide groups, indicative of the chitosan structure, were found at $1647 \mathrm{~cm}^{-1}$, corresponding to the stretching vibration mode of $\mathrm{C}=\mathrm{O}$ (amide I), at $1560 \mathrm{~cm}^{-1}$, related to the stretching vibration mode of $\mathrm{N}-\mathrm{H}$ (amide II), and at $1325 \mathrm{~cm}^{-1}$, attributed to to the vibration of amide III. In the case of the FTIR spectra of the PVP-CS hydrogels with different polymer ratios, it can be seen, as expected, how the signals of the amide groups I $\left(1647 \mathrm{~cm}^{-1}\right)$ and II $\left(1560 \mathrm{~cm}^{-1}\right)$ decrease as the chitosan ratio decreases. Also, a spectrum of the PVP-CS hydrogels shows a peak at $1288 \mathrm{~cm}^{-1}$ characteristic of $\mathrm{C}-\mathrm{N}$ stretching from the pyrrolidone backbone. The $\mathrm{OH}$ stretching absorption around $3300 \mathrm{~cm}^{-1}$ is only due to CS and its broad profile increases with increasing the PVP content. The $v_{\mathrm{OH}}$ band has a constant blue shift, as the PVP concentration increases in the samples. The FTIR spectrum of tetracycline hydrochloride showed the absorption peaks for $\mathrm{N}-\mathrm{H}$ and $\mathrm{O}-\mathrm{H}$ stretching at $3341-3329 \mathrm{~cm}^{-1}$ and aromatic $\mathrm{C}-\mathrm{H}$ stretching at $3085-3024 \mathrm{~cm}^{-1}$. The vibrational peaks at $2995-2863 \mathrm{~cm}^{-1}$ and 1648 $1582 \mathrm{~cm}^{-1}$ were assigned to $\mathrm{C}-\mathrm{H}$ stretching and aromatic $\mathrm{C}=\mathrm{C}$ stretching, respectively. The aromatic in-plane and out-of-plane deformation peaks were observed at $1247-1000 \mathrm{~cm}^{-1}$ and 567 $501 \mathrm{~cm}^{-1}$, respectively. The vibrational peak at $965 \mathrm{~cm}^{-1}$ was assigned to $\mathrm{C}-\mathrm{N}$ stretching. The PVP-CS-TH hydrogels showed absorption peaks for $\mathrm{N}-\mathrm{H}$ and $\mathrm{O}-\mathrm{H}$ stretching at $3413-3325 \mathrm{~cm}^{-1}$ and for aliphatic C-H stretching at $2974-2830 \mathrm{~cm}^{-}$ 1 . From the FTIR analysis, we can observe that the intensity of the absorption band at $965 \mathrm{~cm}^{-1}$ increased in the samples containing $\mathrm{TH}$, when comparing to the control.

The morphology of the freeze-dried hydrogel samples was analyzed by SEM. It was observed that all the freeze-dried samples exhibited 3D porous structures. Also, the SEM images revealed that the morphology and porosity of the PVP-CS hydrogels changed when varying the polymer ratio (Fig. 2 a-c).

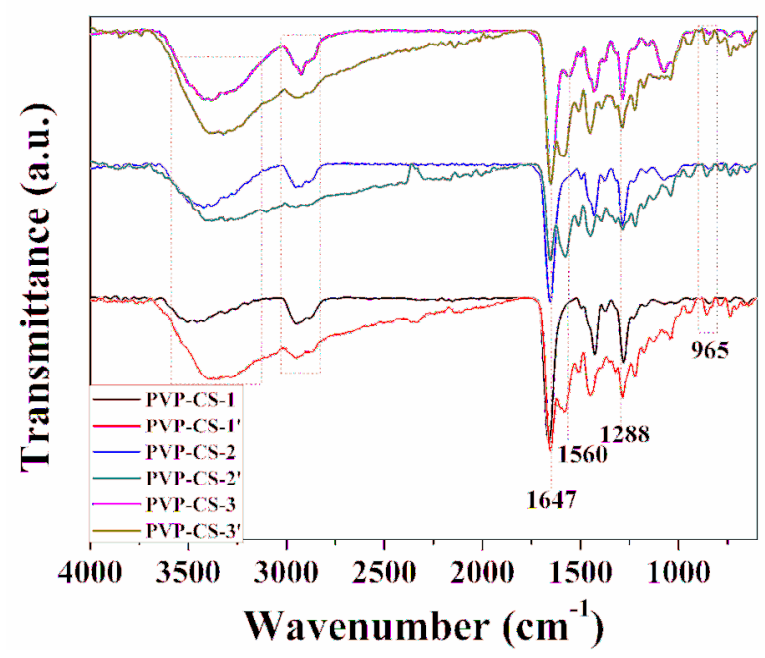

Figure 1: FTIR spectra of PVP-CS and PVP-CS-TH hydrogel 


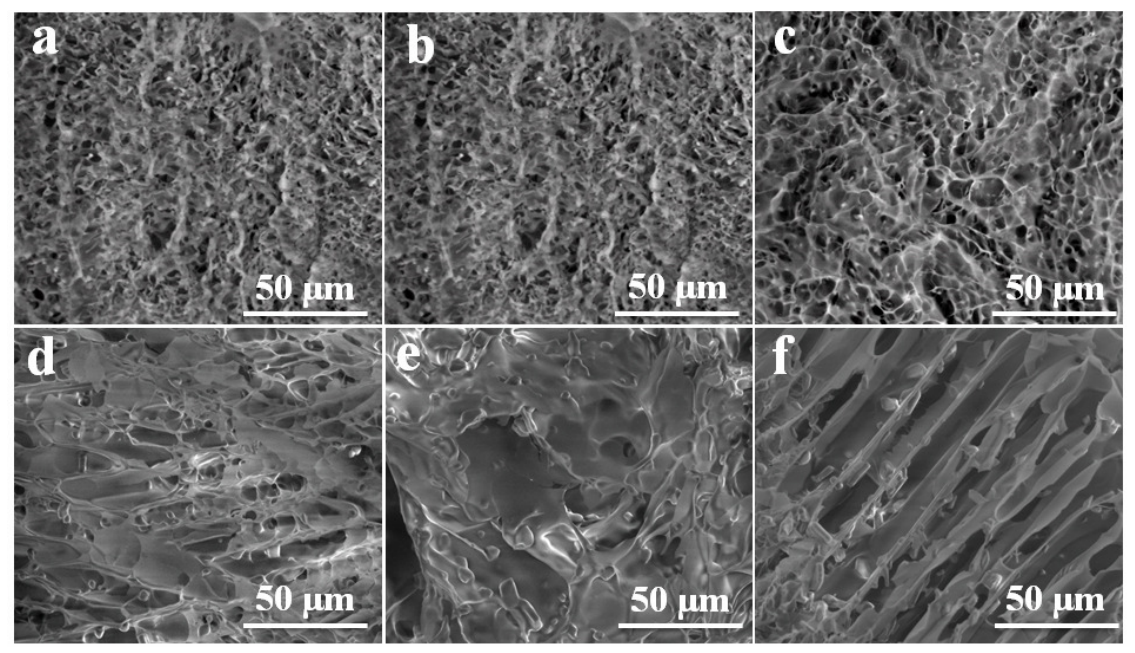

Figure 2: Micrographs of freeze-dried PVP-CS hydrogels: PVP-CS-1 (a), PVP-CS-2 (b), PVP-CS-3 (c) and PVP-CSTH: PVP-CS-1' (d), PVP-CS-2' (e), PVP-CS-3' (f)

The hydrogel with the highest proportion of chitosan (PVP-CS-3) presented the most porous network and the widest pores (Fig. 2 c)..$^{31,32}$ The influence of the drug, $\mathrm{TH}$, on the structure of the hydrogel network was also investigated: Figure 2 (d-f) reveals that tetracycline hydrochloride containing PVP-CS hydrogels were homogeneously loaded. The TH was loaded by first occupying the existing interconnected microporous spaces of the PVP-CS hydrogel network.

\section{Moisture sorption behavior}

The moisture sorption capacity of hydrogels influence their stability, shelf life and medicinal applications. The sorption-desorption isotherms recorded in the dynamic regime, at $25^{\circ} \mathrm{C}$ (Fig. 3), in $10 \%$ humidity steps, revealed that the maximum water vapor sorption capacity increases from $44.32 \%$ to $58.97 \%$ and $59.48 \mathrm{wt} \%$ relative to the dry basis weight of the samples as the initial composition changes, and from $27.83 \%$ to $32.39 \%$ and $38.50 \mathrm{wt} \%$ for the samples containing $\mathrm{TH}$. The highest value of water vapor sorption capacity $(59.48 \%)$ was obtained for the hydrogel with 50\% PVP and 50\% CS (PVP-CS-2) and the lowest value $(27.82 \%)$ was obtained for the tetracycline loaded sample containing 50\% PVP and $50 \%$ CS (PVP-CS-2'). The BrunauerEmmett-Teller (BET) surface area, estimated on the basis of moisture sorption isotherms in the relative humidity range of $0-40 \%$, was between $311.642-363.24 \mathrm{~m}^{2} / \mathrm{g}$ for the drug loaded samples, and 584.9-592.028 $\mathrm{m}^{2} / \mathrm{g}$ for the blank samples.

The type $\mathrm{V}$ sorption/desorption isotherms recorded for all tested samples are associated with the sorption of water vapor on low hydrophilic materials. ${ }^{33-38}$ When the ratio of relative test humidity to initial relative humidity $\mathrm{p} / \mathrm{p}_{0}$ is low ( $\mathrm{RH}<20 \%)$, the type $\mathrm{V}$ isotherm shape can be attributed to relatively weak adsorbent-adsorbate interactions. At higher humidity, molecular clustering is followed by pore filling. This type of isotherm is observed for water adsorption on hydrophobic or less hydrophilic mesoporous adsorbents. The sorption isotherms are S-sigmoid curve, concave on low humidity and convex on high humidity. The sigmoid curve is explained by the model based on Brunauer, Emmett and Teller's multilayer adsorption theory (BET). The water molecules penetrate into the hydrogel matrix, water adsorption occurring not only at the surface, but also inside the hydrogels. For the first layer, based on the BET equation, the water molecules are adsorbed by the polar groups around the polymer chains. If polymers are crosslinked, they cannot be dissolved by water sorption, instead they swell; the numbers of adsorbed layers for this case being limited and polymer solids turn into gel materials. The water sorption capacity of different samples follow a similarly shaped curve (Fig. 4). 


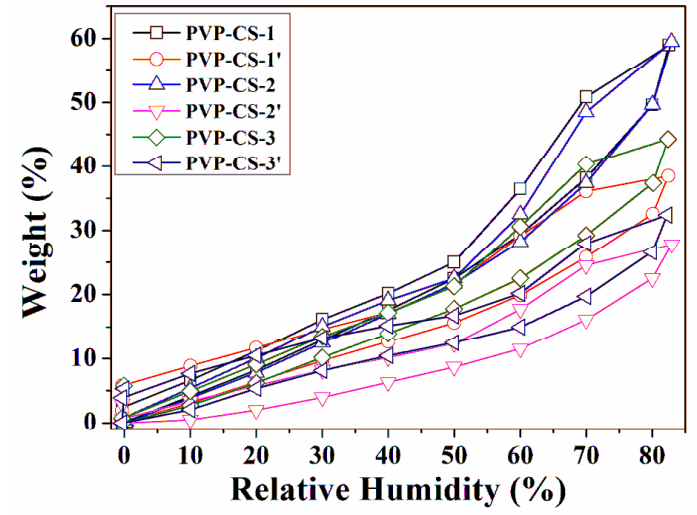

Figure 3: Moisture sorption/desorption isotherms for blank and drug loaded hydrogel samples

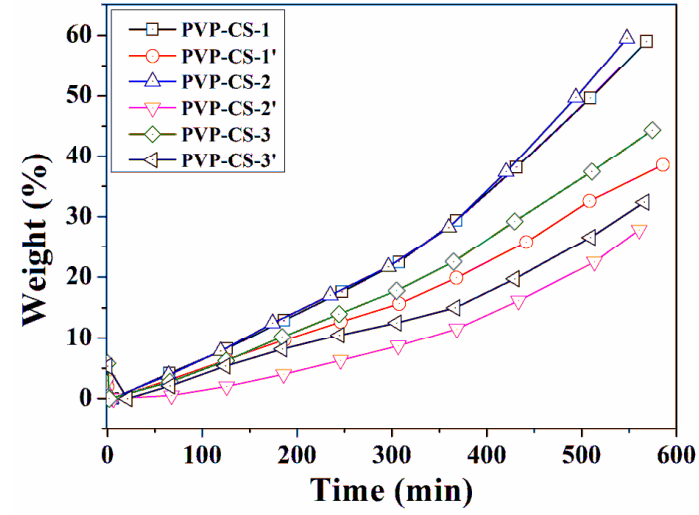

Figure 4: Moisture sorption capacity (relative to dry basis weight of the samples) as a function of time for the studied samples

Table 2

Values of water sorption capacities and BET parameters

\begin{tabular}{|c|c|c|c|c|c|}
\hline \multirow[b]{2}{*}{ Sample } & \multirow{2}{*}{$\begin{array}{l}\text { PVP } \\
(\%)\end{array}$} & \multirow[b]{2}{*}{$\begin{array}{l}\text { CS } \\
(\%)\end{array}$} & \multirow{2}{*}{$\begin{array}{c}\text { Weight } \\
(\%)^{*}\end{array}$} & \multicolumn{2}{|c|}{ BET kinetic model } \\
\hline & & & & $\begin{array}{c}\text { Area } \\
\left(\mathrm{m}^{2} / \mathrm{g}\right)\end{array}$ & $\begin{array}{c}\text { Monolayer } \\
(\mathrm{g} / \mathrm{g})\end{array}$ \\
\hline PVP-CS-1 & 80 & 20 & 58.9741 & 591.252 & 0.1684 \\
\hline PVP-CS-1' & 80 & 20 & 38.5062 & 363.240 & 0.1034 \\
\hline PVP-CS-2 & 50 & 50 & 59.4854 & 584.923 & 0.1666 \\
\hline PVP-CS-2' & 50 & 50 & 27.8261 & 311.642 & 0.0887 \\
\hline PVP-CS-3 & 20 & 80 & 44.3199 & 592.028 & 0.1686 \\
\hline PVP-CS-3' & 20 & 80 & 32.3869 & 325.029 & 0.0926 \\
\hline
\end{tabular}

*relative to dry basis weight of the samples

The values of water vapor sorption capacities for all the samples are presented in Table 2. The isotherms show hysteresis between sorption and desorption. As may be seen in Figure 3, the final mass is not the same as the initial one, because a small part of sorbed water remains in it. The water sorption behavior of these samples was analyzed using moisture sorption isotherms. The role played by water molecules in the sample was interpreted on the basis of the Brunauer-EmmetTeller model (BET) ${ }^{36}$ which allowed a good fit of the water sorption data.

The BET model is the most widely used technique for predicting moisture sorption. It describes the isotherms up to a relative humidity of $50 \%$, depending on the type of sorption isotherm and on the type of material. This model is limited because it cannot completely describe the water sorption behavior of materials in multilayers.

The BET equation (3) is very often used for modeling the sorption isotherms:

$$
W=\frac{W_{m} \cdot C \cdot \frac{p}{p_{0}}}{\left(1-\frac{p}{p_{0}}\right) \cdot\left(1-\frac{p}{p_{0}}+C \cdot \frac{p}{p_{0}}\right)}
$$

where $\mathrm{W}$ - the weight of adsorbed water, $\mathrm{W}_{\mathrm{m}}$ the weight of water forming a monolayer, $\mathrm{C}$ - the sorption constant and $\frac{p}{p_{0}}$ - the relative humidity.

The high hygroscopicity of CS is due to the presence of the pair of free electrons of the amine group, which is supposed to be the origin of the dative bonds. CS absorbed more water because it is more polar. According to the literature, the effect of adsorbed water on hydrophilic materials can be divided into three regions of hydration. For chitosan, when it is hydrated at low values of equilibrium relative humidity $\left(\mathrm{RH}_{\mathrm{eq}}\right)(<50 \%)$, the rate of relaxation of the solvent slightly increases. Intermediate $\mathrm{RH}_{\mathrm{eq}}$ values (from 50 to $75 \%$ ) increase the mobility of the matrix of chitosan. At higher $\mathrm{RH}_{\mathrm{eq}}$ values $(80 \%)$, there is a significant increase in the molecular mobility of the matrix. It has been reported that the chitosan has 3 adsorption sites: the hydroxypropyl group, the 
amino group, and the end of the polymer chain consisting of a hydroxyl or aldehyde group. ${ }^{39,40}$ Based on the degree of interaction between water and polymer, three states of water adsorbed in the hydrophilic materials have been proposed: (i) non-freezable or composition water, which does not crystallize, even at low temperatures, (ii) linked or freezable water, which crystallizes below $0{ }^{\circ} \mathrm{C}$, and (iii) free water, which can be crystallized at $0{ }^{\circ} \mathrm{C}$. After the adsorption of water, chitosan may swell, but will stop to some extent due to the cross-linking of the crystalline zones. ${ }^{40}$ When CS is blended with PVP, the amine and hydroxyl groups of chitosan can interact with the carbonyl groups of PVP, resulting in the formation of a compact three-dimensional (3D) network in the blend and the affinity of chitosan for water is reduced.

The electrostatic interactions and the hydrogen bonding between PVP and chitosan decrease the number of hydrophilic functional groups. During moisture sorption experiments, water molecules can diffuse in, filling up the void volumes, without forming hydrogen bonds. The obtained hydrogels combine the hydrophilic nature of chitosan and PVP with their high affinity for water, thus increasing the water uptake. For the drug loaded samples, the presence of $\mathrm{TH}$, encapsulated in the materials, decrease the moisture sorption capacity as compared with the blank samples, because the $\mathrm{TH}$ first fills the existing interconnected microporous spaces of the PVP-CS hydrogel network. The TH drug loading and, most importantly, its release can be controlled by modifying the ratio of PVP to CS.

\section{Estimation of drug loading and entrapment efficiency}

The drug loading and encapsulation efficiency of the formulations were based on the calibration curve obtained using standard solutions of tetracycline hydrochloride $(5-40 \mu \mathrm{g} / \mathrm{mL})$. The results presented in Table 3 show a direct correlation between drug loading and entrapment efficiency. The entrapment efficiency of the drug in various formulations has been found to be in the range of $79.82-100.97 \%$. From the experimental data, it has been found that, with the increase in the concentration of the cross-linking agent (PVP), there is an increase in the TH load, which is in correlation with the experimental results obtained from the FTIR spectrum.

The IR spectra show the formation of hydrogen bonds between PVP and TH, so that with increasing polymer concentration the efficiency of drug loading also increases.

\section{In vitro drug release}

The results obtained from the in vitro $\mathrm{TH}$ release studies in $\mathrm{pH} 7.4$ buffer media selected for the three formulations are shown in Figure 5. These results show that the combination of the two hydrophilic polymers, PVP-CS-2 (PVP and $\mathrm{CS}$ in a ratio of 50:50) led to a prolonged release system. From the results of the $\mathrm{pH} 7.4$ buffer release test, it has been found that after the first 15 min, the TH released from PVP-CS-3' reaches $30 \%$, from PVP-CS-2' $-27 \%$ and from PVP-CS$1^{\prime}-78 \%$. The drug release rate profile of the PVP-CS hydrogel exhibits a burst release phase, in which $78 \%$ of the loaded TH is released in the first $15 \mathrm{~min}$. The exposure profile of the drug in the PVP-CS sample can be explained by the diffusion of the drug through the crystalline surface of the chitosan structure. After $2 \mathrm{~h}$, the release of tetracycline from the hydrogels was $60 \%, 66 \%$ and $78 \%$, respectively, indicating that drug release from the hydrogels increased with an increase in the PVP concentration of the studied samples. After $6 \mathrm{~h}$, the maximum amount of $\mathrm{TH}$ released from PVP-CS-3' and PVP-CS-1' hydrogels were $75 \%$ and $82 \%$, respectively.

Table 3

Drug loading and encapsulation efficiency profile of studied hydrogels

\begin{tabular}{lccccc}
\hline Sample & $\begin{array}{c}\text { PVP } \\
(\%)\end{array}$ & $\begin{array}{c}\text { CS } \\
(\%)\end{array}$ & $\begin{array}{c}\text { TH } \\
(\%)\end{array}$ & $\begin{array}{c}\text { LC } \pm \text { SD } \\
(\%)\end{array}$ & $\begin{array}{c}\text { EE } \pm \text { SD } \\
(\%)\end{array}$ \\
\hline PVP-CS-1 & 80 & 20 & 3 & $3.03 \pm 0.05$ & $100.97 \pm 1.52$ \\
PVP-CS-2' & 50 & 50 & 3 & $2.39 \pm 0.03$ & $79.82 \pm 1.09$ \\
PVP-CS-3' & 20 & 80 & 3 & $2.79 \pm 0.05$ & $92.95 \pm 1.52$ \\
\hline
\end{tabular}




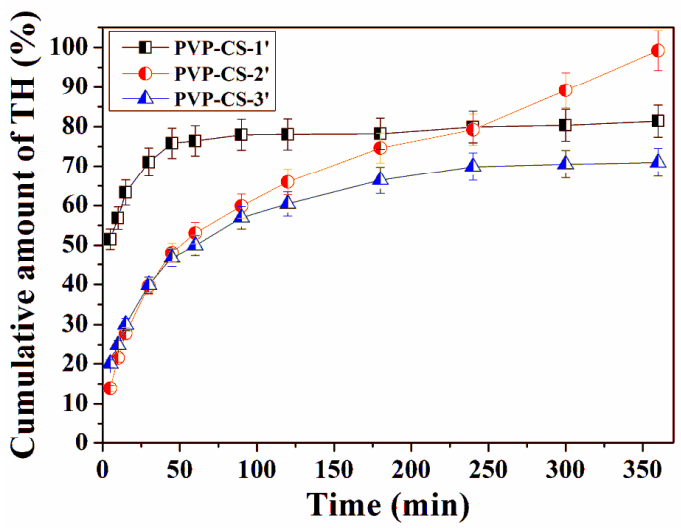

Figure 5: In vitro release profile of $\mathrm{TH}$ from the samples at $\mathrm{pH} 7.4$ and $37{ }^{\circ} \mathrm{C}$

As regards the PVP-CS-2' sample, the maximum amount of tetracycline released over 8 $\mathrm{h}$ was $95 \%$. Thus, the PVP-CS-2' hydrogel can be recommended as a delayed release system for $\mathrm{TH}$. These results are in correlation with the FTIR data, which highlighted the presence of only hydrogen bonds in the formed system. The delayed release of TH from the hydrogels is due to the interactions of the carbonyl groups in the PVP structure with those of the amino and hydroxyl groups in the TH structure, but also of the crystalline areas in the CS structure. Thus, even if PVP has the ability to absorb the drug, it is achieved by hydrogen bonds, which causes a faster release of the drug when in the structure of the hydrogel CS is in a lower percentage (PVPCS-1'). If the concentration of CS is too high, the release is delayed, but not fully achieved, because the release of the drug is prevented by the crystalline areas of the CS. Thus, when there is an optimal ratio between the concentration of PVP and CS (50:50), both substances contribute to the prolonged release of $\mathrm{TH}$.

\section{Analysis of in vitro drug release kinetics}

In order to correlate the data obtained from the in vitro drug release studies with the characteristics of the pharmaceutical form, the kinetic profile of $\mathrm{TH}$ release from the hydrogels was performed. The study was carried out based on four mathematical models: the zero- and firstorder kinetic models, and the Higuchi and the Korsmeyer-Peppas kinetic models. The results obtained were interpreted by the value of the correlation coefficient $\left(r^{2}\right)$. The highest degree of the correlation coefficient determines the appropriate mathematical model that describes the release kinetics of $\mathrm{TH}^{41}$ Table 4 illustrates the regression coefficients $\left(\mathrm{R}^{2}\right)$ and the calculated equation constants ( $\mathrm{K}, \mathrm{AIC}$ and $\mathrm{n}$ ).

During the drug release studies, there was no surface erosion of the PVP-CS-2' sample, while there was slight degradation of the PVP-CS-3' and PVP-CS- $1^{\prime}$ samples. The in vitro release behavior of the TH-loaded hydrogels was fitted to the first-order kinetic model in order to assess its sustained release potential at the mucosal level. ${ }^{42}$ In addition, the Higuchi model, considered as an important element in the development of drug delivery systems with a homogeneous and granular matrix, was also used to investigate the dissolution and diffusion-dependent drug release profile of the drug carriers. ${ }^{43}$ The KorsmeyerPeppas model is typically applied to polymeric systems and defines the exponential release of the active ingredient over time. As can be seen from Table 4, the $\mathrm{R}^{2}$ and AIC values indicate that not all the formulations follow zero- and first-order kinetics.

For PVP-CS-2', it has been found that the value of the correlation coefficient is higher, therefore, we can interpret that the main mechanism of TH release is diffusion controlled. From the graphical representation of the cumulative percentage of $\mathrm{TH}$ release over time, it may be noted that the drug release from the PVPCS-2' matrix perfectly follows the Higuchi drug release pattern, because there is a direct correlation between the drug release profile and the surface of the hydrogel, very close to the value of the correlation coefficient $\left(\mathrm{r}^{2}=\right.$ 0.9480). ${ }^{43,44}$ In the case of the Korsmeyer-Peppas model, much higher values of $\mathrm{R}^{2}$ (between 0.9740-0.9903) were obtained, and the lowest values for AIC suggested that this model fits best the release data. The obtained results, presented in 
Table 4, reveal that the release of $\mathrm{TH}$ from all the studied systems is facilitated by diffusion, as the release data fit perfectly with the KorsmeyerPeppas model.

Table 4

Curve fitting results of in vitro $\mathrm{TH}$ release profile of hydrogels

\begin{tabular}{|c|c|c|c|c|}
\hline \multirow{2}{*}{ Kinetic model } & \multirow{2}{*}{ Model coefficients } & \multicolumn{3}{|c|}{ Sample } \\
\hline & & PVP-CS-1' & PVP-CS-2' & PVP-CS-3' \\
\hline \multirow{3}{*}{ Zero-order } & $\mathrm{K}_{0}(\mu \mathrm{g} / \mathrm{h})$ & 20.3456 & 20.4374 & 16.7984 \\
\hline & $\mathrm{R}^{2}$ & 0.2921 & 0.8389 & 0.6812 \\
\hline & AIC & 100.4513 & 82.5360 & 85.1953 \\
\hline \multirow{3}{*}{ First-order } & $\mathrm{K}_{1}\left(\mathrm{~h}^{-1}\right)$ & 3.8472 & 0.6631 & 0.4894 \\
\hline & $\mathrm{R}^{2}$ & 0.2855 & 0.9066 & 0.5065 \\
\hline & AIC & 77.2645 & 58.8643 & 72.7304 \\
\hline \multirow{3}{*}{ Higuchi } & $\mathrm{K}_{\mathrm{h}}\left(\mathrm{h}^{0.5}\right)$ & 47.0828 & 43.1185 & 39.9689 \\
\hline & $\mathrm{R}^{2}$ & 0.4011 & 0.9480 & 0.7289 \\
\hline & AIC & 88.9906 & 51.1497 & 64.9442 \\
\hline \multirow{4}{*}{ Korsmeyer-Peppas } & $\mathrm{n}$ & 0.09 & 0.38 & 0.26 \\
\hline & $\mathrm{K}_{\mathrm{p}}\left(\mathrm{h}^{-\mathrm{n}}\right)$ & 71.8718 & 49.4786 & 47.6079 \\
\hline & $\mathrm{R}^{2}$ & 20.3456 & 0.9903 & 0.9802 \\
\hline & AIC & 0.2921 & 31.3405 & 34.4429 \\
\hline
\end{tabular}

The values of the $n$ exponential coefficient in the Korsmeyer-Peppas equation $(\mathrm{n}<0.5)$, as well as the minimum values of AIC, confirmed the release of TH through a diffusion process. This type of diffusion is characteristic of semi-solid pharmaceutical forms and polymeric films, for which the rate of release of the drug substance is proportional to the square root of time. ${ }^{43,45}$

Because during the release tests, no change was found on the surface of sample PVP-CS-2', while for samples PVP-CS-3' and PVP-CS-1', a slight degradation of the hydrogels was observed, the zero-order kinetic model was applied. ${ }^{27}$ Because the TH-loaded hydrogels were developed to obtain modified-release pharmaceutical forms to be administered at the mucosal level, it was necessary to apply the first-order kinetic model as well. ${ }^{44}$ However, the Korsmeyer-Peppas model is superior $\left(\mathrm{R}^{2}>0.9903\right)$ to the Higuchi, zero- and first-order kinetic models, in describing the release kinetics of TH. The $n$ values of the Korsmeyer-Peppas model are mostly less than 0.5 , suggesting the release mechanism was governed by diffusion. This type of diffusion is characteristic of semi-solid pharmaceutical forms and polymeric films, for which the rate of release of the drug substance is proportional to the square root of time. ${ }^{45}$

\section{CONCLUSION}

Tetracycline loaded PVP-CS hydrogels were successfully prepared using the freeze-drying technique, with various proportions of the component materials mixed to obtain three formulations of hydrogels. The PVP-CS hydrogels displayed microporous structures. Morphological studies clearly showed good dispersion of TH. The highest value of water vapor sorption capacity was obtained for the hydrogel with 50\% PVP and 50\% CS (PVP-CS2 ), while the lowest value was recorded for the drug loaded sample (PVP-CS-2'). The TH release profile can be controlled by modifying the ratio of PVP to CS. Furthermore, the diffusion-controlled release of TH recommends the PVP-CS-2 hydrogel to be used as a controlled release system in the treatment of bacterial infections and oral candidiasis.

Based on the data obtained for the release of $\mathrm{TH}$, the PVP-CS-2 hydrogel is considered to be the best candidate to be used in pharmaceutical formulations for the local treatment of bacterial infections and oral candidiasis.

ACKNOWLEDGEMENTS: The authors acknowledge the financial support of CNCSISUEFISCSU, Project Number PN-III-P1-1.1-TE2019-0639 nr. 89/03.09.2020.

\section{REFERENCES}

1 M. Teodorescu and M. Bercea, Polym.-Plast. Technol., $\quad \mathbf{5 4}, \quad 923 \quad$ (2015), https://doi.org/10.1080/03602559.2014.979506

2 W. Wessel, M. Schoog and E. Winkler, Arzneimittel-Forschung, 21, 1468 (1971)

3 P. Franco and I. De Marco, Polymers, 12, 1114 (2020), https://doi.org/10.3390/polym12051114 
4 M. Kurakula and G. S. N. K. Rao, J. Drug Deliv. Sci. Technol., $\quad$ 60, $102046 \quad$ (2020), https://doi.org/10.1016/j.jddst.2020.102046

5 M. Dash, F. Chiellini, R. M. Ottenbrite and E. Chiellini, Prog. Polym. Sci., 36, 981 (2011), https://doi.org/10.1016/j.progpolymsci.2011.02.001

6 S. Aihua, X. Dai and Z. Jing, Polym. Sci., Series A, 62, 228

(2020),

https://doi.org/10.1134/S0965545X20030128

7 R. H. Sizílio, J. G. Galvão, G. G. G. Trindade, L. T. S. Pina, L. N. Andrade et al., Carbohyd. Polym., 190, 339

https://doi.org/10.1016/j.carbpol.2018.02.079

(2018),

8 R. Kumar, S. Ranwa and G. Kumar, J. Phys. Chem.

B., 124, $149 \quad$ (2020),

https://doi.org/10.1021/acs.jpcb.9b08897

9 E. Marsano, S. Vicini, J. Skopiǹska, M. Wisniewski and A. Sionkowska, Macromol. Symp., 218, 251 (2004), https://doi.org/10.1002/masy.200451426

10 J.-T. Yeh, C.-L. Chen, K. S. Huang, Y. H. Nien, J. L. Chen et al., J. Appl. Polym. Sci., 101, 885 (2006), https://doi.org/10.1002/app.23517

11 A. Sosnik and K. P. Seremeta, Gels, 3, 25 (2017), https://doi.org/10.3390/gels3030025

12 L. A. Sharpe, A. M. Daily, S. D. Horava and N. A. Peppas, Expert Opin. Drug Del., 11, 901 (2014), https://doi.org/10.1517/17425247.2014.902047

13 A. K. Gaharwar, N. A. Peppas and A. Khademhosseini, Biotechnol. Bioeng., 111, 441 (2014), https://doi.org/10.1002/bit.25160

14 A. Concheiro and C. Alvarez-Lorenzo, Adv. Drug Deliv. Rev., $\quad$ 65, $1188 \quad$ (2013), https://doi.org/10.1016/j.addr.2013.04.015

15 B. D. Ratner, A. S. Hoffman and E. A. F. J. Schoen, "Biomaterials Science: An Introduction to Materials in Medicine", Academic Press, San Diego, CA, 2004, vol. 2

16 S. Li, S. Dong, W. Xu, S. Tu, L. Yan et al., Adv. Sci. (Weinh), 5, $1700527 \quad$ (2018), https://doi.org/10.1002/advs.201700527

17 T. Vermonden, R. Censi and W. E. Hennink, Chem.

Rev., 112, 2853 (2012),

https://doi.org/10.1021/cr200157d

18 K. Jepsen and S. Jepsen, Periodontology 2000, 71, 82 (2016), https://doi.org/10.1111/prd.12121

19 A. Scribante, P. K. Vallittu and M. Özcan, Biomed. Res. Int., 2018, $4734986 \quad$ (2018), https://doi.org/10.1155/2018/4734986

20 A. Kapoor, R. Malhotra, V. Grover and D. Grover, Dent. Res. J. (Isfahan), 9, 505 (2012), https://doi.org/10.4103/1735-3327.104866

21 T. Phaechamud and J. Charoenteeraboon, AAPS PharmSciTech, 9, $829 \quad$ (2008), https://doi.org/10.1208/s12249-008-9117-x

22 S. R. Shah, A. M. Henslee, P. P. Spicer, S. Yokota, S. Petrichenko et al., Pharm. Res., 31, 3379 (2014), https://doi.org/10.1007/s11095-014-1427-y
23 M. O. Griffin, E. Fricovsky, G. Ceballos and F. Villarreal, Am. J. Physiol. Cell Physiol., 299, C539 (2010), https://doi.org/10.1152/ajpcell.00047.2010

24 G. Schenk, T. F. Flemmig, T. Betz, J. Reuther and B. Klaiber, Clin. Oral Implants Res., 8, 427 (1997), https://doi.org/10.1034/j.1600-0501.1997.080510.x

25 D. S. Jones, M. S. Lawlor and A. D. Woolfson, Curr. Drug Deliv., 1, 17 (2004), https://doi.org/10.2174/1567201043480027

${ }^{26}$ H. Yilmaz Atay, in "Functional Chitosan", edited by S. Jana, Springer, Singapore, 2020, pp. 457-489, https://doi.org/10.1007/978-981-15-0263-7_15

27 M. R. Marques, R. Loebenberg and M. Almukainzi, Dissolut. Technol., $\quad$ 18, $15 \quad$ (2011), http://dx.doi.org/10.14227/DT180311P15

28 M. Gohel, K. Sarvaiya, A. Shah and B. Brahmbhatt, Indian J. Pharm. Sci., 71, 142 (2009), https://doi.org/10.4103/0250-474X.54281

29 B. Glaessl, F. Siepmann, I. Tucker, T. Rades and J. Siepmann, J. Drug Deliv. Sci. Technol., 20, 127 (2010), https://doi.org/10.1016/S1773-2247(10)500170

30 A. I. Romero, M. Villegas, A. G. Cid, M. L. Parentis, E. E. Gonzo et al., Asian J. Pharm. Sci., 13, 54 (2018), https://doi.org/10.1016/j.ajps.2017.08.007

31 M. D. Figueroa-Pizano, I. Vélaz, F. J. Peñas, P. Zavala-Rivera, A. J. Rosas-Durazo et al., Carbohyd. Polym., $\quad$ 195, $476 \quad$ (2018), https://doi.org/10.1016/j.carbpol.2018.05.004

32 J. H. Sung, M.-R. Hwang, J. O. Kim, J. H. Lee, Y. I. Kim et al., Int. J. Pharm., 392, 232 (2010), https://doi.org/10.1016/j.ijpharm.2010.03.024

33 IUPAC Recommendations, Pure Appl. Chem., 66, 1739 (1994), http://dx.doi.org/10.1351/pac199466081739

34 IUPAC Recommendations Pure Appl. Chem., 57, 603 (1985),

http://dx.doi.org/10.1351/pac198557040603

35 M. Thommes, K. Kaneko, A. V. Neimark, J. P. Olivier, F. Rodriguez-Reinoso et al., Pure Appl. Chem., 87, 1051 (2015), https://doi.org/10.1515/pac2014-1117

36 C. Buttersack, Phys. Chem. Chem. Phys., 21, 5614 (2019), https://doi.org/10.1039/C8CP07751G

37 A. Nistor, G. Stiubianu, C. Racles and M. Cazacu, Materiale Plastice, 48, 33 (2011)

38 T. Vlad-Bubulac, D. Serbezeanu, A.-M. Oprea, I.D. Carja, C. Hamciuc et al., Cent. Eur. J. Chem., 11, 446 (2013), https://doi.org/10.2478/s11532-012-01743

39 M. F. Cervera, J. Heinämäki, M. Räsänen, S. Maunu, M. Karjalainen et al., Carbohyd. Polym., 58, 401

(2004),

https://doi.org/10.1016/j.carbpol.2004.08.017

40 H. Gocho, H. Shimizu, A. Tanioka, T.-J. Chou and T. Nakajima, Carbohyd. Polym., 41, 87 (2000), https://doi.org/10.1016/S0144-8617(99)00113-7 


\section{Chitosan}

41 A. Rescign, "Foundation of Pharmacokinetics", Kluwer Academic/Plenum Publishers, New York, 2003

${ }^{42}$ H. Shaikh, R. V. Kshirsagar and S. G. Patil, World J. Pharm. Pharm. Sci., 4, 324 (2015)

43 R. Gouda, H. Baishya and Z. Qing, J. Dev. Drug., 6, (2017), https://doi.org/10.4172/2329-6631.1000171
44 T. Vlad-Bubulac, A.-M. Oprea, D. Serbezeanu, I.D. Carja and C. Hamciuc, Rev. Chim. (Bucharest), 64, 663 (2013)

45 F. Brandl, F. Kastner, R. M. Gschwind, T. Blunk, J. Teßmar et al., J. Control. Release, 142, 221 (2010), https://doi.org/10.1016/j.jconrel.2009.10.030 